\title{
Chronic Pancreatitis Finding by Endoscopic Ultrasonography in the Pancreatic Parenchyma of Intraductal Papillary Mucinous Neoplasms Is Associated with Invasive Intraductal Papillary Mucinous Carcinoma
}

\author{
Mamoru Takenaka a, e Atsuhiro Masuda ${ }^{a}$ Hideyuki Shiomi ${ }^{a}$ Yosuke Yagi ${ }^{a}$ \\ Yoh Zen $^{\text {b }}$ Arata Sakai $^{a}$ Takashi Kobayashi $^{a}$ Yoshifumi Arisaka ${ }^{a}$

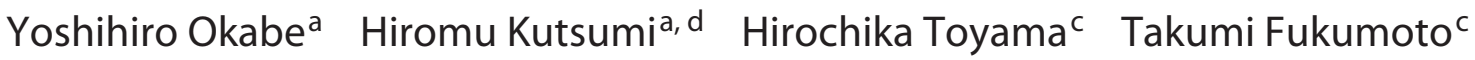 \\ Yonson Kuc Masatoshi Kudo ${ }^{\mathrm{e}}$ Takeshi Azuma ${ }^{\mathrm{a}}$ \\ a Division of Gastroenterology, Department of Internal Medicine, ${ }^{b}$ Division of Diagnostic Pathology, and \\ 'Division of Hepato-Biliary-Pancreatic Surgery, Department of Surgery, Kobe University Graduate School of \\ Medicine, ${ }^{\mathrm{d}}$ Center for Clinical Research and Advanced Medicine Establishment, Shiga University of Medical Science, \\ Kobe, and ${ }^{\mathrm{e}}$ Department of Gastroenterology and Hepatology, Kindai University Graduate School of Medicine, \\ Osaka-Sayama, Japan
}

\section{Keywords}

Chronic pancreatitis · Endoscopic ultrasonography . Intraductal papillary mucinous neoplasm · Pancreatic parenchyma

\begin{abstract}
Background/Objectives: The recent guideline for intraductal papillary mucinous neoplasms (IPMNs) focuses on morphological features of the lesion as signs of malignant transformation, but ignores the background pancreatic parenchyma, including features of chronic pancreatitis (CP), which is a risk factor for pancreatic malignancies. Endoscopic ultrasonography frequently reveals evidence of CP (EUS-CP findings) in the background pancreatic parenchyma of patients with IPMNs. Therefore, we investigated whether background EUS-CP findings were associated with malignant IPMN.
\end{abstract}

\section{KARGER}

(C) 2017 S. Karger AG, Basel

E-Mail karger@karger.com

www.karger.com/ocl
Methods: The clinical data of 69 consecutive patients with IPMNs who underwent preoperative EUS and surgical resection between April 2010 and October 2014 were collected prospectively. The association of EUS-CP findings (total number of EUS-CP findings; 0 vs. $\geq 1$ ) with invasive IPMN was examined. The association of EUS-CP findings with pathological changes of the background pancreatic parenchyma (atrophy/inflammation/fibrosis) was also examined. Results: Among patients with EUS-CP findings, invasive intraductal papillary mucinous carcinoma (IPMC) was significantly more frequent than among patients without EUS-CP findings (42.5\% [17/40] vs. $3.4 \%[1 / 29], p=0.0002)$. In addition, patients with EUS-CP findings had higher grades of pancreatic

M. Takenaka, A. Masuda, and H. Shiomi contributed equally to this work. 
atrophy and inflammation than patients without EUS-CP findings (atrophy: $72.5 \%$ [29/40] vs. $34.5 \%$ [10/29], $p=0.003$; inflammation: $45.0 \%[18 / 40]$ vs. $20.7 \%$ [6/29], $p=0.04)$. Conclusions: In IPMN patients, detection of EUS-CP findings in the background pancreatic parenchyma was associated with a higher prevalence of invasive IPMC. Accordingly, EUS examination should not only assess the morphological features of the lesion itself, but also EUS-CP findings in the background parenchyma.

(c) 2017 S. Karger AG, Basel

\section{Introduction}

Intraductal papillary mucinous neoplasms (IPMNs) are a well-characterized group of intraductal mucin-producing cystic neoplasms of the pancreas with malignant potential $[1,2]$. In the 2012 international consensus guidelines, high-risk IPMN included two clinical categories with "high-risk stigmata" and "worrisome features," and different therapeutic strategies were recommended for both groups. It is recommended that patients with "highrisk stigmata" be treated by surgical resection [3-7]. Patients who have "worrisome features" and additional high-risk signs should also undergo resection, while patients with "worrisome features" alone should receive careful follow-up. The "high-risk stigmata" of IPMNs that can be detected by endoscopic ultrasonography (EUS) are an enhancing solid component inside the cyst and dilation of the main pancreatic duct $(>10 \mathrm{~mm})$ [3, 8-11]. Additional high-risk signs among the "worrisome features" are a definite mural nodule and suspected main duct involvement $[12,13]$. Thus, the current guidelines focus on the morphological features of IPMN as revealed by EUS.

On the other hand, although not described in previous reports, clinical experience suggests that evidence of chronic pancreatitis (CP) is frequently identified in the background pancreatic parenchyma of IPMN patients by EUS (EUS-CP findings). CP is a well-known risk factor for pancreatic malignancy, including pancreatic ductal adenocarcinoma [14-16]. However, the relationship between malignant transformation of IPMNs and pathological changes of the pancreatic parenchyma, such as atrophy, inflammation, and fibrosis, remains unclear. Moreover, changes of the background parenchyma are not considered to be a high-risk sign for malignant IPMN in the current guideline. We hypothesized that EUS-CP findings might be associated with malignant IPMN.

To test this hypothesis, we utilized a database of 69 consecutive patients with IPMNs who underwent preopera- tive EUS and surgical resection at Kobe University Hospital. We examined the association of EUS-CP findings with the incidence of malignant IPMN and with pathological changes of the background parenchyma in the resected specimens (atrophy, inflammation, and fibrosis).

\section{Methods}

\section{Study Design}

The clinical data of 69 consecutive patients with IPMNs in whom EUS was performed before surgical resection at Kobe University Hospital from April 2010 to October 2014 were collected prospectively. Patients with concomitant pancreatic carcinoma (defined by the presence of two discrete tumors) were excluded. We investigated various clinical and tumor characteristics, including age, sex, abdominal symptoms (presence vs. absence), serum CEA ( $<5$ vs. $\geq 5 \mathrm{ng} / \mathrm{mL}$ ), serum CA19-9 (<37 vs. $\geq 37 \mathrm{U} / \mathrm{mL}$ ), diabetes mellitus (presence vs. absence), habitual drinking (none/ sometimes/daily), alcohol consumption ( $<50$ vs. $\geq 50$ g/day), macroscopic type of IPMN (main duct, mixed, or branch duct type), cyst location (head vs. body/tail), main pancreatic duct diameter $(<5,5-10,>10 \mathrm{~mm})$, mural nodule in the cyst (presence vs. absence), cyst diameter $(<30,30-40,>40 \mathrm{~mm})$, and tumor mucin phenotype (intestinal, gastric, pancreatobiliary, or oncocytic). According to the 2012 international consensus guidelines [3], the macroscopic type of IPMN was classified as main duct type (MDIPMN), mixed type (Mix-IPMN), or branch duct type (BD-IPMN) based on the results of imaging studies, mainly magnetic resonance cholangiopancreatography (MRCP). Tumors were histologically classified into four types (gastric, intestinal, pancreatobiliary, or oncocytic) according to the appearance on hematoxylin and eosin-stained sections and immunohistochemistry with antimucin antibodies (MUC1/MUC2/MUC5AC/MUC6). In patients with multiple morphologically distinct IPMNs, each neoplasm was classified depending on the type of the dominant component. Tumors were also classified into low-grade dysplasia, high-grade dysplasia, or invasive intraductal papillary mucinous carcinoma (IPMC). The diameter of the main pancreatic duct and the cyst size were measured by MRCP in most cases. In patients who did not undergo MRCP, measurement was done by an alternative method, such as endoscopic retrograde cholangiopancreatography or computed tomography. The presence of mural nodules was evaluated by EUS and magnetic resonance imaging. Abdominal symptoms were defined as including abdominal or back pain/discomfort, weight loss, appetite loss, and jaundice.

This study was conducted in accordance with the Declaration of Helsinki and its amendments (UMIN-CTR ID: 000021345). The study protocol was approved by the ethics committee of Kobe University School of Medicine (No. 1864). All authors had access to the study data and reviewed and approved the final manuscript.

\section{Evaluation of the Pancreatic Parenchyma by EUS}

Three echo-endoscopes (GF-UCT240, GF-UCT260, or GFUE260; Olympus, Tokyo, Japan) were used in this study. EUS-CP findings refer to the parenchymal features of CP in the Rosemont classification recently proposed as EUS diagnostic criteria for this condition [17]. These findings included "hyperechoic foci with
62

Oncology 2017;93(suppl 1):61-68 DOI: $10.1159 / 000481232$
Takenaka et al. 

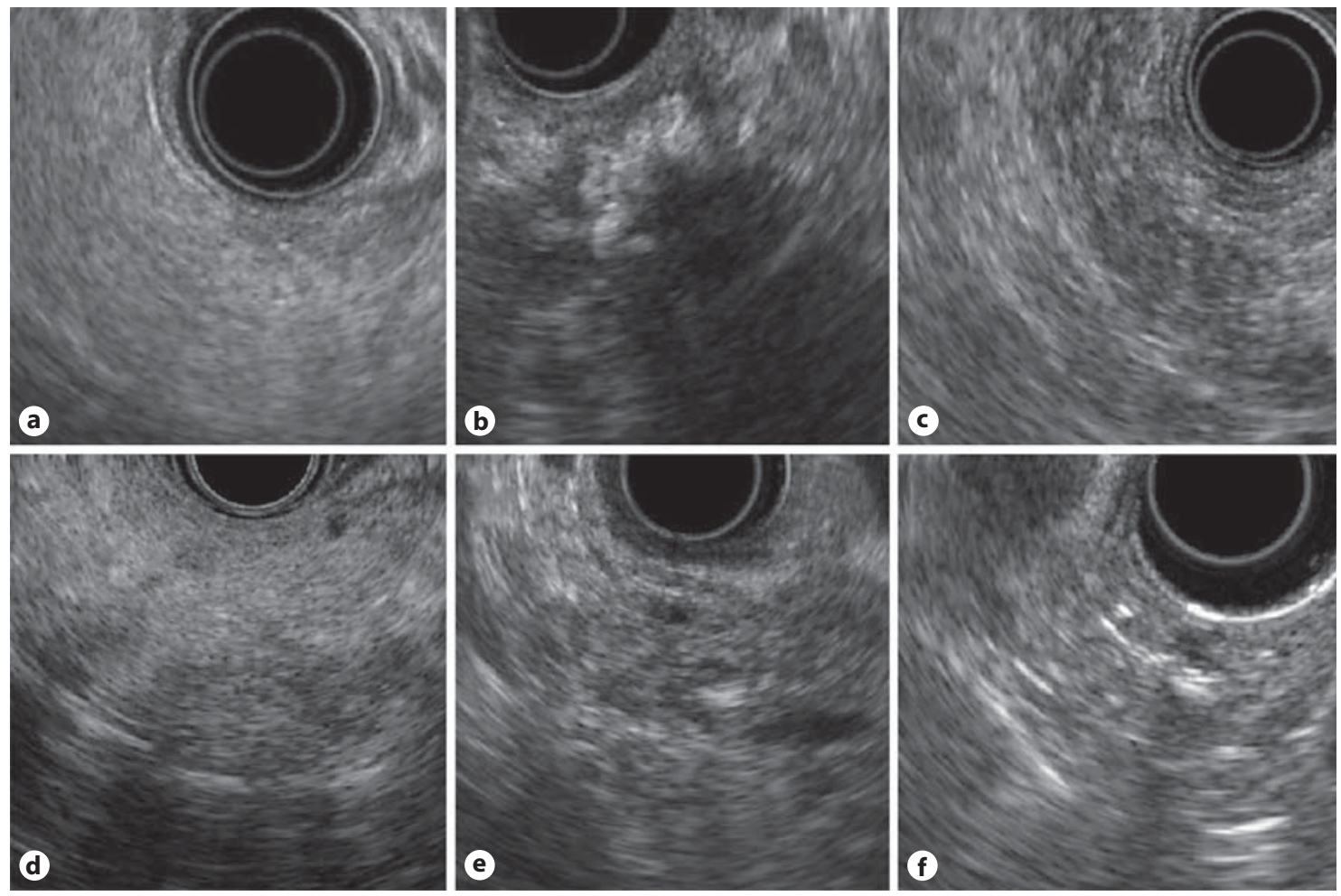

Fig. 1. Typical EUS-CP findings based on the parenchymal features of CP according to the Rosemont classification. a Normal. b Hyperechoic foci with shadowing. c Lobularity with honeycombing. d Lobularity without honeycombing. e Hyperechoic foci without shadowing. f Stranding. CP, chronic pancreatitis; EUS, endoscopic ultrasonography.

shadowing," "lobularity with honeycombing," "lobularity without honeycombing," "hyperechoic foci without shadowing," and "stranding" (Fig. 1). "Cysts" is also a parenchymal feature of CP in the Rosemont classification, but it was excluded because IPMN itself affects cyst formation. For the same reason, ductal features of $\mathrm{CP}$ in the Rosemont classification were excluded, including "main pancreatic duct calculi," "irregular main pancreatic duct contour," "dilated side branches," "main pancreatic duct dilation," and "hyperechoic main pancreatic duct margin."

EUS-CP findings were reviewed prospectively by an endosonographer (M. Takenaka) who was unaware of the other data. All cases were re-examined by a second endosonographer $(H$. Shiomi), who was also unaware of the other data, and there was a good correlation of EUS-CP findings between the two endosonographers $(\kappa=0.80$ for score 0 vs. $\geq 1, p<0.001)$. Both endosonographers had experience with more than 1,000 EUS examinations.

\section{Indications for Surgical Resection}

Indications for surgical resection of IPMN at our hospital were decided according to the 2012 International Consensus Guidelines. According to the guidelines, resection is recommended for MD-IPMN. In the case of Mix-IPMN and BD-IPMN, resection is recommended if the patient has "high-risk stigmata." In patients with "worrisome features" and additional high-risk signs, resection is also recommended.

Chronic Pancreatitis Imaging by EUS in the Pancreatic Parenchyma of IPMNs
Pathological Evaluation of Pancreatic Parenchymal Atrophy, Inflammation, and Fibrosis

Hematoxylin and eosin-stained tissue sections from all resected specimens of IPMN were reviewed by a pathologist (Y. Zen), who was blinded to all clinical features. Pancreatic parenchymal atrophy, infiltration of inflammatory cells, and fibrosis were evaluated in the background pancreatic parenchyma. The severity of parenchymal atrophy, inflammation, or fibrosis was graded as 0 (none), $1+$ (mild), $2+$ (moderate), or $3+$ (severe). Representative examples are shown in Figure 2.

\section{Statistical Analysis}

All statistical analyses were conducted using JMP version 11 (SAS Institute, Cary, NC, USA), and all $p$ values were two-sided. The $\chi^{2}$ test (or Fisher exact test, if appropriate) was used to assess associations between categorical variables. To assess the association of the number of EUS-CP findings ( $0 \mathrm{vs} . \geq 1$ ) with the incidence of invasive IPMN, we conducted multivariate logistic regression analysis. The binary categorical variable (presence and absence) of the incidence of invasive IPMN was used as an outcome variable. Multivariate binary logistic regression analysis was performed to adjust for potential confounders. The OR was adjusted for age, alcohol consumption, and macroscopic type of IPMN, the $p$ value of which was $<0.2$ in univariate analysis. Kappa coefficients were calculated to assess agreement of EUS-CP findings between the two observers. In all analyses, $p<0.05$ was considered statistically significant.

Oncology 2017;93(suppl 1):61-68 DOI: $10.1159 / 000481232$ 

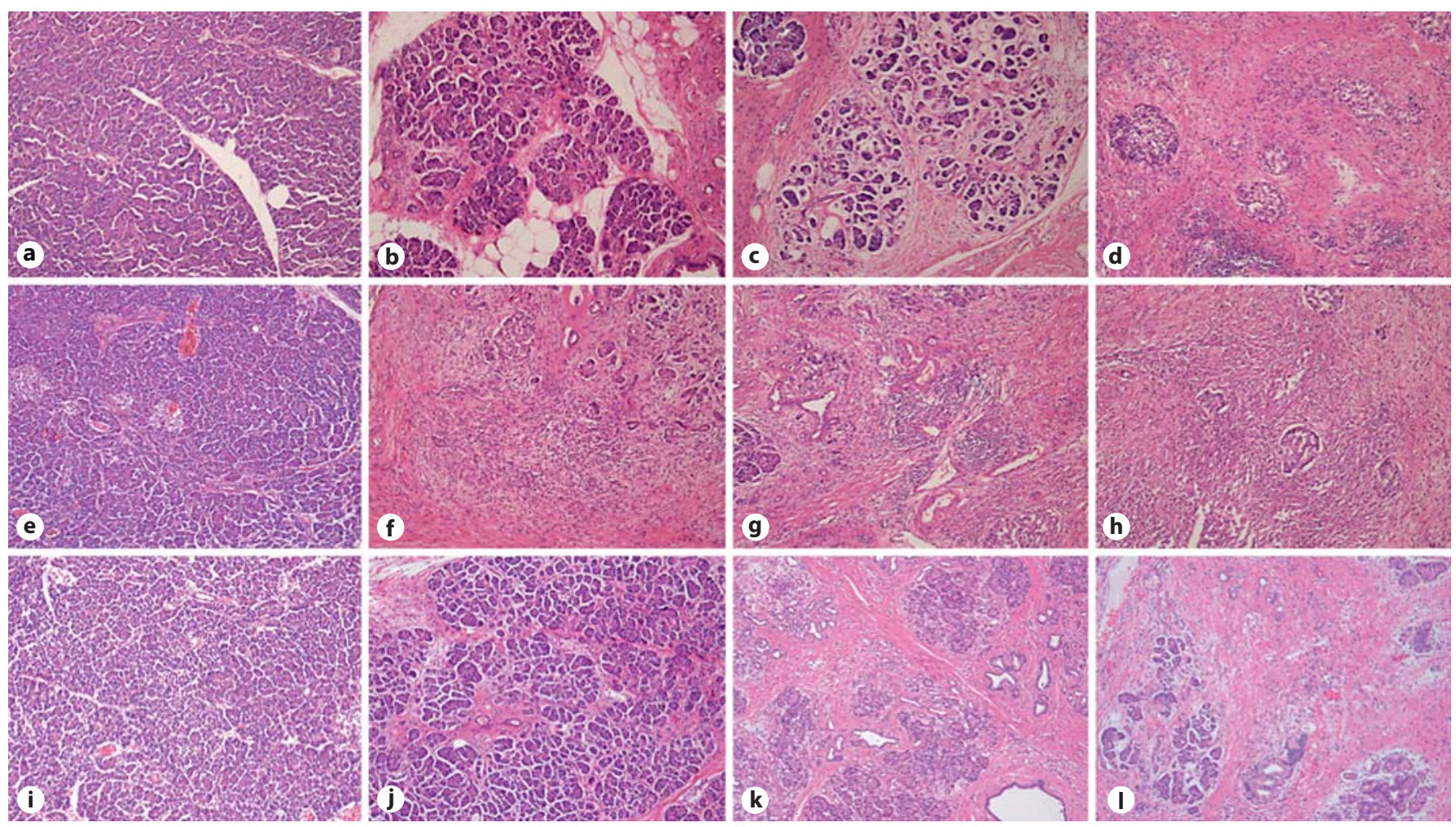

Fig. 2. Pathological findings of the background pancreas parenchyma in intraductal papillary mucinous neoplasm patients. The figure shows the grading of parenchymal atrophy, inflammation, and fibrosis of the pancreas. a-d Parenchymal atrophy of grades $0-3$. a Grade 0 (no atrophic change). b Grade 1 (focal atrophy). c Grade 2 (moderate atrophy). d Grade 3 (severe atrophy with few

residual acinar cells). e-h Inflammation of grades $0-3$. e Grade 0 (no inflammation). $\mathbf{f}$ Grade 1 (mild inflammation). g Grade 2 (moderate inflammation with lymphoid aggregates). h Grade 3 (massive inflammation), i-I Fibrosis of grades $0-3$. i Grade 0 (no fibrosis). j Grade 1 (mild fibrosis). k Grade 2 (moderate fibrosis). I Grade 3 (massive fibrosis).

\section{Results}

Association of EUS-CP Findings with Invasive IPMC

We categorized 69 IPMN patients by clinical features and factors associated with EUS-CP findings. There were 29 patients without EUS-CP findings and 40 patients with at least one EUS-CP finding. The clinical and morphological features of IPMN are summarized with respect to EUS-CP findings in Table 1. Patients with EUSCP findings were older than those without EUS-CP findings (70.2 \pm 1.4 vs. $65.0 \pm 1.7$ years, $p=0.02)$. Among the patients with EUS-CP findings, invasive IPMC was significantly more frequent than among those without EUS$\mathrm{CP}$ findings (42.5\% [17/40] vs. $3.4 \%[1 / 29], p=0.0002)$ (Table 2). Multivariate analysis showed that detection of EUS-CP findings in the background pancreatic parenchyma was associated with a higher prevalence of invasive IPMC (multivariate OR $=21.9,95 \% \mathrm{CI}=3.8-423.1$,

$p=0.0001)$. Assessment of each EUS-CP finding showed that invasive IPMC was significantly more frequent in the patients with "stranding" than in those without (55\% [11/20] vs. $14 \%$ [7/49], $p=0.002)$. In addition, invasive IPMC was significantly more frequent in the patients who had "lobularity without honeycombing" than in those without (83\% [5/6] vs. $21 \%$ [13/63], $p=0.004)(\mathrm{Ta}-$ ble 3).

\section{Association of EUS-CP Findings with Pathological \\ Changes of the Pancreatic Parenchyma}

Table 4 shows the association between the total number of EUS-CP findings and the pathological findings (atrophy/inflammation/fibrosis) in the background pancreatic parenchyma of the IPMN patients. Among patients with EUS-CP findings, higher grades of pancreatic atrophy and inflammation were observed than in those without EUS-CP findings (atrophy: 72.5\% [29/40]

Takenaka et al. 
Table 1. Characteristics of the patients and the IPMNs (number of EUS-CP findings 0 vs. $\geq 1$ )

\begin{tabular}{|c|c|c|c|}
\hline & \multicolumn{2}{|c|}{ Number of EUS-CP findings } & \multirow[t]{2}{*}{$p$ value } \\
\hline & 0 & $\geq 1$ & \\
\hline Patients, $n$ & 29 & 40 & \\
\hline Age, years & $65.0 \pm 1.7$ & $70.2 \pm 1.4$ & 0.02 \\
\hline Sex, male/female & $17 / 12$ & $20 / 20$ & 0.50 \\
\hline Abdominal symptoms & $5(17 \%)$ & $11(28 \%)$ & 0.32 \\
\hline $\mathrm{CEA} \geq 5 \mathrm{ng} / \mathrm{mL}$ & $8(28 \%)$ & $13(33 \%)$ & 0.66 \\
\hline CA19-9 $\geq 37 \mathrm{U} / \mathrm{mL}$ & $8(28 \%)$ & $7(18 \%)$ & 0.32 \\
\hline Diabetes mellitus & $10(35 \%)$ & $13(33 \%)$ & 0.86 \\
\hline Habitual drinking (none/sometimes/daily) & $14 / 7 / 8$ & $22 / 9 / 9$ & 0.84 \\
\hline Alcohol consumption $\geq 50 \mathrm{~g} /$ day & $1(3 \%)$ & $5(13 \%)$ & 0.19 \\
\hline \multicolumn{4}{|l|}{ Clinical features of IPMN } \\
\hline Macroscopic type (main duct/mixed/branch duct) & $0 / 16 / 13$ & $5 / 24 / 11$ & 0.08 \\
\hline Location of cyst (head/body-tail) & $18 / 11$ & $24 / 16$ & 0.86 \\
\hline Diameter of main pancreatic duct $(<5 / 5-10 / \geq 10 \mathrm{~mm})$ & $12 / 10 / 7$ & $10 / 14 / 16$ & 0.26 \\
\hline Mural nodule in cyst & $11(38 \%)$ & $18(45 \%)$ & 0.56 \\
\hline Cyst size $(<30 / 30-40 / \geq 40 \mathrm{~mm})$ & $14 / 8 / 7$ & $23 / 3 / 14$ & 0.08 \\
\hline Mucin phenotype (intestinal/gastric/pancreatobiliary/oncocytic) & $8 / 16 / 4 / 1$ & $12 / 24 / 4 / 0$ & 0.64 \\
\hline Invasive IPMC & $1(3 \%)$ & $17(43 \%)$ & 0.0002 \\
\hline
\end{tabular}

Values are presented as $n, n(\%)$, or mean \pm SD. $p<0.05$ was considered statistically significant. CP, chronic pancreatitis; EUS, endoscopic ultrasonography; IPMC, intraductal papillary mucinous carcinoma; IPMN, intraductal papillary mucinous neoplasm.

Table 2. Frequency of invasive IPMC in relation to the number of EUS-CP findings

\begin{tabular}{|c|c|c|c|c|}
\hline \multirow{2}{*}{$\begin{array}{l}\text { Univariate } \\
\text { analysis }\end{array}$} & \multirow{2}{*}{$\begin{array}{l}\text { Patients, } \\
n\end{array}$} & \multicolumn{2}{|c|}{ Invasive IPMC } & \multirow{2}{*}{$\begin{array}{l}p \\
\text { value }\end{array}$} \\
\hline & & present & absent & \\
\hline \multicolumn{5}{|l|}{ EUS-CP findings } \\
\hline$\geq 1$ & 40 & $17(43 \%)$ & $23(57 \%)$ & \multirow{2}{*}{0.0002} \\
\hline 0 & 29 & $1(3 \%)$ & $28(97 \%)$ & \\
\hline $\begin{array}{l}\text { Multivariate } \\
\text { analysis }\end{array}$ & \multicolumn{3}{|c|}{ 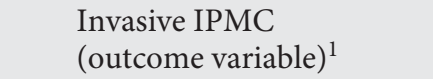 } & $\begin{array}{l}p \\
\text { value }\end{array}$ \\
\hline EUS-CP findings $(\geq 1)$ & \multicolumn{3}{|c|}{$21.9(3.8-423.1)$} & 0.0001 \\
\hline
\end{tabular}

$p<0.05$ was considered statistically significant. ${ }^{1}$ Presented as OR (95\% CI). The score presents as follows: 0 (absent), 1+ (present). The OR was adjusted for age, alcohol consumption, macroscopic type of IPMN, of which $p$ was $<0.2$ in Table 1 (univariate analysis). $\mathrm{CP}$, chronic pancreatitis; EUS, endoscopic ultrasonography; IPMC, intraductal papillary mucinous carcinoma.

Chronic Pancreatitis Imaging by EUS in the Pancreatic Parenchyma of IPMNs
Table 3. Prevalence of invasive IPMC in relation to each EUS-CP finding

\begin{tabular}{|c|c|c|c|c|}
\hline & \multirow{2}{*}{$\begin{array}{l}\text { Patients, } \\
n\end{array}$} & \multicolumn{2}{|c|}{ Invasive IPMC } & \multirow{2}{*}{$\begin{array}{l}p \\
\text { value }\end{array}$} \\
\hline & & present & absent & \\
\hline Total & 69 & $18(26 \%)$ & $51(74 \%)$ & \\
\hline \multicolumn{5}{|c|}{ Hyperechoic foci with shadowing } \\
\hline Present & 10 & $4(40 \%)$ & $6(60 \%)$ & \multirow{2}{*}{0.44} \\
\hline Absent & 59 & $14(24 \%)$ & $45(76 \%)$ & \\
\hline \multicolumn{5}{|c|}{ Lobularity with honeycombing } \\
\hline Present & 2 & $1(50 \%)$ & $1(50 \%)$ & \multirow{2}{*}{0.46} \\
\hline Absent & 67 & $17(25 \%)$ & $50(75 \%)$ & \\
\hline \multicolumn{5}{|c|}{ Lobularity without honeycombing } \\
\hline Present & 6 & $5(83 \%)$ & $1(17 \%)$ & \multirow{2}{*}{0.004} \\
\hline Absent & 63 & $13(21 \%)$ & $50(79 \%)$ & \\
\hline \multicolumn{5}{|c|}{ Hyperechoic foci without shadowing } \\
\hline Present & 27 & $10(37 \%)$ & $17(63 \%)$ & \multirow{2}{*}{0.16} \\
\hline Absent & 42 & $8(19 \%)$ & $34(81 \%)$ & \\
\hline \multicolumn{5}{|l|}{ Stranding } \\
\hline Present & 20 & $11(55 \%)$ & $9(45 \%)$ & \multirow{2}{*}{0.002} \\
\hline Absent & 49 & $7(14 \%)$ & $42(86 \%)$ & \\
\hline
\end{tabular}

$p<0.05$ was considered statistically significant. $\mathrm{CP}$, chronic pancreatitis; EUS, endoscopic ultrasonography; IPMC, intraductal papillary mucinous carcinoma. 
Table 4. Association between the number of EUS-CP findings and pathological changes in the background pancreatic parenchyma of IPMN patients

\begin{tabular}{|c|c|c|c|c|c|c|c|c|c|c|}
\hline & \multirow{2}{*}{$\begin{array}{l}\text { Patients, } \\
n\end{array}$} & \multicolumn{2}{|l|}{ Atrophy } & \multirow{2}{*}{$\begin{array}{l}p \\
\text { value }\end{array}$} & \multicolumn{2}{|c|}{ Inflammation } & \multirow{2}{*}{$\begin{array}{l}p \\
\text { value }\end{array}$} & \multicolumn{2}{|l|}{ Fibrosis } & \multirow{2}{*}{$\begin{array}{l}p \\
\text { value }\end{array}$} \\
\hline & & high & low & & high & low & & high & low & \\
\hline Total & 69 & $39(57 \%)$ & $30(43 \%)$ & & $24(57 \%)$ & $45(43 \%)$ & & $32(46 \%)$ & $37(54 \%)$ & \\
\hline \multicolumn{11}{|c|}{ Total number of EUS-CP findings } \\
\hline$\geq 1$ & 40 & $29(73 \%)$ & $11(27 \%)$ & \multirow{2}{*}{0.003} & $18(45 \%)$ & $22(55 \%)$ & \multirow{2}{*}{0.04} & $21(53 \%)$ & $19(47 \%)$ & \multirow{2}{*}{0.33} \\
\hline 0 & 29 & $10(34 \%)$ & $19(66 \%)$ & & $6(21 \%)$ & $23(79 \%)$ & & $11(38 \%)$ & $18(62 \%)$ & \\
\hline
\end{tabular}

Atrophy, inflammation, and fibrosis were divided into two groups based on the grade of pathological findings (high, moderate, or severe; low, none, or mild). $p<0.05$ was considered statistically significant. CP, chronic pancreatitis; EUS, endoscopic ultrasonography; IPMN, intraductal papillary mucinous neoplasm.

Table 5. Association between each EUS-CP finding and pathological changes in the background pancreatic parenchyma of IPMN patients

\begin{tabular}{|c|c|c|c|c|c|c|c|c|c|c|}
\hline & \multirow{2}{*}{$\begin{array}{l}\text { Patients, } \\
n\end{array}$} & \multicolumn{2}{|l|}{ Atrophy } & \multirow{2}{*}{$\begin{array}{l}p \\
\text { value }\end{array}$} & \multicolumn{2}{|c|}{ Inflammation } & \multirow{2}{*}{$\begin{array}{l}p \\
\text { value }\end{array}$} & \multicolumn{2}{|l|}{ Fibrosis } & \multirow{2}{*}{$\begin{array}{l}p \\
\text { value }\end{array}$} \\
\hline & & high & low & & high & low & & high & low & \\
\hline Total & 69 & $39(57 \%)$ & $30(43 \%)$ & & $24(57 \%)$ & $45(43 \%)$ & & $32(46 \%)$ & $37(54 \%)$ & \\
\hline \multicolumn{11}{|c|}{ Hyperechoic foci with shadowing } \\
\hline Present & 10 & $8(80 \%)$ & $2(20 \%)$ & \multirow{2}{*}{0.17} & $6(60 \%)$ & $4(40 \%)$ & \multirow{2}{*}{0.09} & $6(60 \%)$ & $4(40 \%)$ & \multirow{2}{*}{0.50} \\
\hline Absent & 59 & $31(53 \%)$ & $28(47 \%)$ & & $18(31 \%)$ & $41(69 \%)$ & & $26(44 \%)$ & $33(56 \%)$ & \\
\hline \multicolumn{11}{|c|}{ Lobularity with honeycombing } \\
\hline Present & 2 & $2(100 \%)$ & $0(0 \%)$ & \multirow[b]{2}{*}{0.50} & $0(0 \%)$ & $2(100 \%)$ & \multirow[b]{2}{*}{0.54} & $2(100 \%)$ & $0(0 \%)$ & \multirow{2}{*}{0.21} \\
\hline Absent & 67 & $37(55 \%)$ & $30(45 \%)$ & & $24(36 \%)$ & $43(64 \%)$ & & $30(45 \%)$ & $37(55 \%)$ & \\
\hline \multicolumn{11}{|c|}{ Lobularity without honeycombing } \\
\hline Present & 6 & $5(83 \%)$ & $1(17 \%)$ & \multirow{2}{*}{0.22} & $4(67 \%)$ & $2(33 \%)$ & \multirow[b]{2}{*}{0.17} & $5(83 \%)$ & $1(17 \%)$ & \multirow{2}{*}{0.09} \\
\hline Absent & 63 & $34(54 \%)$ & $29(46 \%)$ & & $20(32 \%)$ & $43(68 \%)$ & & $27(43 \%)$ & $36(57 \%)$ & \\
\hline \multicolumn{11}{|c|}{ Hyperechoic foci without shadowing } \\
\hline Present & 27 & $18(67 \%)$ & $9(33 \%)$ & \multirow{2}{*}{0.22} & $11(41 \%)$ & $16(59 \%)$ & \multirow{2}{*}{0.45} & $11(41 \%)$ & $16(50 \%)$ & \multirow{2}{*}{0.47} \\
\hline Absent & 42 & $21(50 \%)$ & $21(50 \%)$ & & $13(31 \%)$ & $29(69 \%)$ & & $21(50 \%)$ & $21(50 \%)$ & \\
\hline \multicolumn{11}{|l|}{ Stranding } \\
\hline Present & 20 & $18(90 \%)$ & $2(10 \%)$ & \multirow{2}{*}{0.0004} & $11(55 \%)$ & $9(45 \%)$ & \multirow{2}{*}{0.03} & $15(75 \%)$ & $5(25 \%)$ & \multirow{2}{*}{0.003} \\
\hline Absent & 49 & $21(59 \%)$ & $28(41 \%)$ & & $13(27 \%)$ & $36(73 \%)$ & & $17(35 \%)$ & $32(65 \%)$ & \\
\hline
\end{tabular}

Atrophy, inflammation, and fibrosis were divided into two groups based on the grade of pathological findings (high, moderate to severe; low, none to mild). $p<0.05$ was considered statistically significant. CP, chronic pancreatitis; EUS, endoscopic ultrasonography; IPMN, intraductal papillary mucinous neoplasm.

vs. $34.5 \%$ [10/29], $p=0.003$; inflammation: $45.0 \%$ [18/40] vs. $20.7 \%$ [6/29], $p=0.04)$. Assessment of the pathological correlates with each EUS-CP finding revealed higher grades of pancreatic atrophy, inflammation, and fibrosis in patients with "stranding" than in those without (atrophy: $90 \%[18 / 20]$ vs. $43 \%$ [21/49], $p=$ 0.0004; inflammation: $56 \%$ [11/20] vs. $27 \%$ [13/49], $p=$ 0.03 ; fibrosis: $75 \%$ [15/20] vs. $35 \%$ [17/49], $p=0.003$ ) (Table 5).
Association of Pathological Changes in the Pancreatic Parenchyma with Invasive IPMC

Figure 3 displays the relationship between pathological changes (atrophy, inflammation, and fibrosis) in the background pancreatic parenchyma and invasive IPMC. Higher grades of atrophy and inflammation were significantly associated with the presence of invasive IPMC (atrophy: $p=0.002$; inflammation: $p=0.002$ ). Higher grades of fibrosis were also associated with invasive IPMC, but the relationship was not significant $(p=0.057)$. 


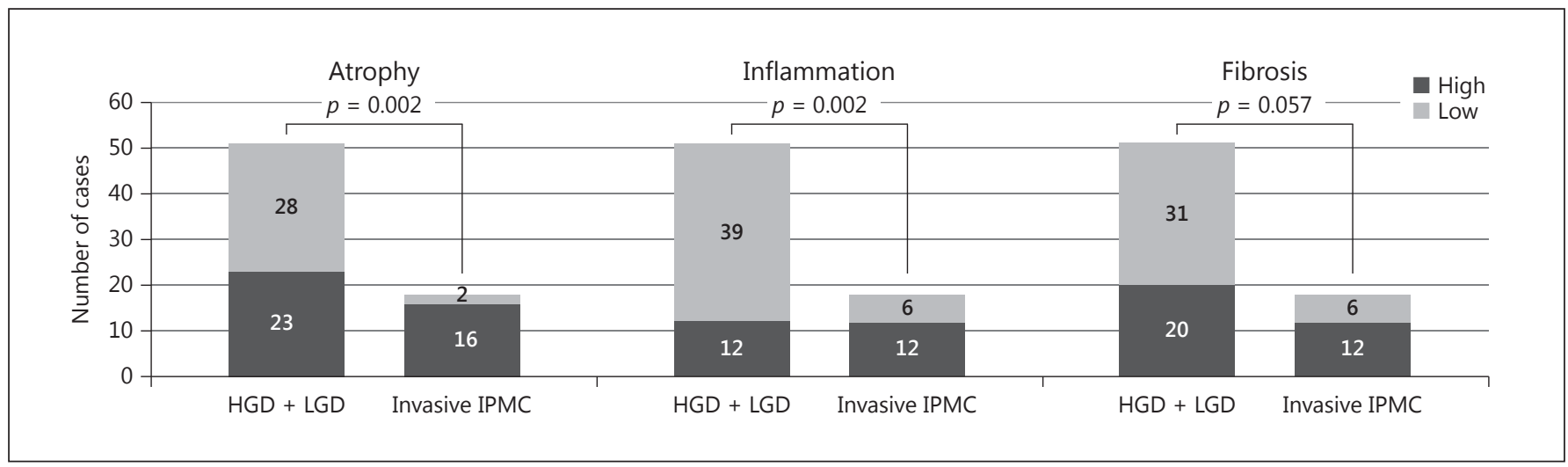

Fig. 3. Association of pathological changes in the pancreatic parenchyma of intraductal papillary mucinous neoplasm with invasive intraductal papillary mucinous carcinoma (IPMC). The figure displays the relationship between pathological changes (atrophy, inflammation, and fibrosis) in the background pancreatic parenchy- ma and invasive IPMC. $p<0.05$ was considered statistically significant. Atrophy, inflammation, and fibrosis were divided into two groups based on the grade of pathological findings (high, moderate to severe; low, none to mild). HGD, high-grade dysplasia; LGD, low-grade dysplasia.

\section{Discussion}

In this study, we found that the frequency of invasive IPMC was significantly higher in patients with EUS-CP findings than in those without. In other words, IPMN patients with "normal background pancreatic parenchyma" on EUS had a significantly lower rate of malignancy. To the best of our knowledge, this is the first study to examine the association between EUS-CP findings and malignant transformation of IPMNs.

The Rosemont classification has been proposed as EUS criteria for diagnosing CP [17]. The EUS-CP findings used in this study corresponded to the parenchymal features of the Rosemont classification. However, the relationship between EUS findings and pathological findings in patients with CP has not been well characterized, even in the Rosemont classification. One of the reasons for this is that tissue specimens for evaluating the pancreatic parenchyma often cannot be obtained in patients with $\mathrm{CP}$ because they rarely undergo surgery. In this study, we were able to examine the relationship between EUS-CP findings and pathological changes of the background pancreatic parenchyma because we used the resected specimens from IPMN patients.

In the Rosemont classification, each EUS finding has a predictive value, and the findings are categorized into major or minor criteria. The major criteria of CP are "hyperechoic foci with shadowing" and "lobularity with honeycombing." On the other hand, "stranding" and "lobularity without honeycombing" are classified as minor cri-

Chronic Pancreatitis Imaging by EUS in the Pancreatic Parenchyma of IPMNs teria because of poor specificity. In the present study, "stranding" and "lobularity without honeycombing" were frequently observed in the background parenchyma of IPMN patients, which was a discrepancy between our results and the Rosemont classification. This difference might have arisen because the causes of CP were different. The Rosemont classification was mainly based on alcoholic CP. On the other hand, our study did not target alcoholic CP, but instead assessed CP caused by mucin stagnation in IPMN patients. It has been reported that hypersecretion of mucin by IPMNs causes obstruction of the main pancreatic duct, which may induce low-grade pancreatitis [2]. This difference in the pathogenesis of the parenchymal changes might be associated with the difference of major EUS findings.

Alcohol consumption is strongly associated with CP [18-20]. In our study, there were no significant differences in alcohol consumption between the groups with and without EUS-CP findings, but the presence of these findings was associated with pathological changes of the background parenchyma, such as atrophy and inflammation. This indicates that the EUS-CP findings we investigated were not associated with alcohol consumption and that an increase in mucin production with malignant transformation of IPMN might be associated with changes in the background parenchyma.

The present study has some limitations. First, it was difficult to demonstrate the association of each EUS-CP finding with specific pathological features. Even in the Rosemont classification, only "hyperechoic foci with

Oncology 2017;93(suppl 1):61-68 DOI: $10.1159 / 000481232$ 
shadowing" and "cysts" (excluded from this study) have histological correlates such as calcification and pseudocyst, while other factors (including "stranding" and "lobularity with honeycombing") are not associated with specific histological features. Further studies will be required to clarify the pathological correlates of these findings. Next, this was a retrospective single-center study, and the number of subjects was limited. Thus, larger studies will be necessary to confirm our results.

In conclusion, detection of EUS-CP findings in the pancreatic parenchyma of IPMN patients was associated with invasive IPMC. When EUS examination of IPMN is performed, the background parenchyma should be evaluated in addition to the morphological features of the tumor itself.

\section{Acknowledgments}

We would like to thank the staff of the Center of Clinical Research (Dr. Omori) at Kobe University Hospital for their valuable contributions (advice about statistical analysis). The authors assume full responsibility for the analysis and interpretation of the data in this report.

This work was supported by grants from JSPS KAKENHI (Grants-in-Aid for Scientific Research; grant No. 15624848 to A. Masuda and grant No. 15612795 to H. Kutsumi). This work was also supported by the Research Committee of Intractable Pancreatic Diseases from the Ministry of Health, Labor, and Welfare of Japan (A. Masuda, H. Shiomi, and H. Kutsumi).

\section{Disclosure Statement}

The authors have no conflicts of interest to declare.

\section{References}

1 Retter J, Dinter D, Bersch C, Singer MV, Lohr M: Acute recurrent pancreatitis curtaining an intraductal papillary mucinous tumor of the pancreas. J Gastrointestin Liver Dis 2007;16: 445-447.

2 Kloppel G: Clinicopathologic view of intraductal papillary-mucinous tumor of the pancreas. Hepatogastroenterology 1998; 45: 1981-1985.

3 Tanaka M, Fernandez-del Castillo C, Adsay $\mathrm{V}$, et al: International consensus guidelines 2012 for the management of IPMN and MCN of the pancreas. Pancreatology 2012;12:183197.

4 Mimura T, Masuda A, Matsumoto I, et al: Predictors of malignant intraductal papillary mucinous neoplasm of the pancreas. J Clin Gastroenterol 2010;44:e224-e229.

5 Crippa S, Fernandez-Del Castillo C, Salvia R, et al: Mucin-producing neoplasms of the pancreas: an analysis of distinguishing clinical and epidemiologic characteristics. Clin Gastroenterol Hepatol 2010;8:213-219.

6 Suzuki Y, Atomi Y, Sugiyama M, et al: Cystic neoplasm of the pancreas: a Japanese multiinstitutional study of intraductal papillary mucinous tumor and mucinous cystic tumor. Pancreas 2004;28:241-246.
7 Rodriguez JR, Salvia R, Crippa S, et al: Branchduct intraductal papillary mucinous neoplasms: observations in 145 patients who underwent resection. Gastroenterology 2007; 133:72-79; quiz 309-310.

8 Aso T, Ohtsuka T, Matsunaga T, et al: "Highrisk stigmata" of the 2012 international consensus guidelines correlate with the malignant grade of branch duct intraductal papillary mucinous neoplasms of the pancreas. Pancreas 2014;43:1239-1243.

9 Ohno E, Hirooka Y, Itoh A, et al: Intraductal papillary mucinous neoplasms of the pancreas: differentiation of malignant and benign tumors by endoscopic ultrasound findings of mural nodules. Ann Surg 2009;249:628-634.

10 Buscaglia JM, Shin EJ, Giday SA, et al: Awareness of guidelines and trends in the management of suspected pancreatic cystic neoplasms: survey results among general gastroenterologists and EUS specialists. Gastrointest Endosc 2009;69:813-820; quiz 820.e1-e17.

11 Ridtitid W, DeWitt JM, Schmidt CM, et al: Management of branch-duct intraductal papillary mucinous neoplasms: a large singlecenter study to assess predictors of malignancy and long-term outcomes. Gastrointest Endosc 2016;84:436-445.

12 Yamamoto N, Kato H, Tomoda T, et al: Contrast-enhanced harmonic endoscopic ultrasonography with time-intensity curve analysis for intraductal papillary mucinous neoplasms of the pancreas. Endoscopy 2016;48:26-34.
13 Kitano M, Kamata K, Imai H, et al: Contrastenhanced harmonic endoscopic ultrasonography for pancreatobiliary diseases. Dig Endosc 2015;27(suppl 1):60-67.

14 Lowenfels AB, Maisonneuve P, Cavallini G, et al: Pancreatitis and the risk of pancreatic cancer. International Pancreatitis Study Group. N Engl J Med 1993;328:1433-1437.

15 Talamini G, Falconi M, Bassi C, et al: Incidence of cancer in the course of chronic pancreatitis. Am J Gastroenterol 1999;94:12531260.

16 Malka D, Hammel P, Maire F, et al: Risk of pancreatic adenocarcinoma in chronic pancreatitis. Gut 2002;51:849-852.

17 Catalano MF, Sahai A, Levy M, et al: EUSbased criteria for the diagnosis of chronic pancreatitis: the Rosemont classification. Gastrointest Endosc 2009;69:1251-1261.

18 Majumder S, Chari ST: Chronic pancreatitis. Lancet 2016;387:1957-1966.

19 Kume K, Masamune A, Ariga H, Shimosegawa T: Alcohol consumption and the risk for developing pancreatitis: a case-control study in Japan. Pancreas 2015;44:53-58.

20 Ye W, Lagergren J, Weiderpass E, Nyren O, Adami HO, Ekbom A: Alcohol abuse and the risk of pancreatic cancer. Gut 2002;51:236239. 